\title{
"Ich warte und warte, jeden Tag."- Fallbeispiel Nr. 3: Maryam Y. (36)
}

Mitte 2017 begegne ich Maryam Y. inmitten einer Gruppe von Geflüchteten wieder. Alle warten gespannt auf ihren Termin im Landesamt Berlin. Zu diesem Zeitpunkt ist sie 36 Jahre alt und bereits seit gut einem Jahr in Deutschland. Ich traf sie bereits einige Male kurz im medizinischen Zentrum, als sie Medikamente für ihren Sohn (12) benötigte. Die dort anwesenden Asylbewerber*innen tragen in ihren Händen Plastiktüten, gefüllt mit Blättern, Attesten, Formularen und Aktenordnern. Nach etlichen Stunden der Warterei außerhalb des Gebäudes kommen sie kohortenweise in die Räumlichkeiten des Landesamts. Sie sitzen auf grauen, festgeschraubten Stühlen und warten den ganzen Tag. Meist vergeblich, denn am Ende des Tages werden sie nach Hause geschickt, um am nächsten Morgen mithilfe eines Zettels, den sie noch erhalten, schneller an die Reihe zu kommen. Während andere sich lautstark unterhalten, in ihre Mobiltelefone sprechen oder sich sonst hörbar mit ihnen beschäftigen, fällt Maryam mit ihrer Schweigsamkeit auf, während sie wartet. Täglich häufen sich unbearbeitete Fälle im Landesamt für Flüchtlingsangelegenheiten, was zu Frust und Missgunst auf allen Seiten führt. Eines der Hauptthemen unter Asylbewerber*innen im Feld ist daher die permanente Zeitverschwendung aufgrund der Wartezeiten. Am Anfang meiner Forschung erschien mir diese Feststellung eher trivial, doch wurde sie zunehmend zu einem unverkennbaren Kennzeichen der Institution Asyl und entsprechend bedeutsam für die Untersuchung von Emotionen im Asylprozess. Die Wartezeiten bilden aber auch eine ideale Gelegenheit, um Kontakte herzustellen, alltägliche Gespräche zu führen und Einblicke in die verschiedenen Lebenswelten zu erhalten. Nachdem ich Maryam von meinem Forschungsprojekt berichtet und sie gefragt habe, ob sie einverstanden sei, sich mehrere Male interviewen zu lassen, erkundigt sie sich nach meinem Projekt und willigt am selben Tag ein. Unsere vier Treffen finden innerhalb von sieben Wochen im Spätsommer 2017 
statt. Einmal treffen wir uns an ihrem Heim, ansonsten warten wir gemeinsam am Landesamt. In der Summe kommen gut fünf Stunden Interviewzeit zusammen.

Für Maryam wird das tägliche Warten zur Pein. Erst nach mehrmaligem Nachfragen, wie es ihr in Berlin geht, kommen wir ins Gespräch.

„Ich warte und warte, jeden Tag. Ich bin wirklich fertig, ich kann einfach nicht mehr. Jeden Tag muss ich hin und her laufen, von der einen Behörde zur nächsten, und dann abermals ewig warten. Ich hätte mir nie im Leben vorgestellt, dass in deutschen Behörden solch ein Chaos herrscht. Außerdem könnte ich in der Zeit so viele sinnvolle Dinge tun und mein Leben in Deutschland vorbereiten. Sprachkurse zum Beispiel. Sie glauben ja gar nicht, was für ein Gefühl das ist, endlich lernen zu dürfen, ohne ein schlechtes Gewissen zu bekommen. Aber jetzt bin ich abgelehnt worden und habe zusätzlich den Trennungsstress am Hals. Dass das Leben in Deutschland so ist, hätte bei Gott keiner von uns gedacht, aber (...), naja ich bin trotzdem dankbar. Außerdem muss ich zum Psychiater, um mir attestieren zu lassen, dass ich ernsthafte psychische Probleme habe. Wegen der Abschiebung. Das sagt jedenfalls mein Anwalt. Ich habe das Gefühl, mich im Kreis zu drehen und langsam tatsächlich verrückt zu werden. Warten auf Termine, Sprachkurse, Gerichtsurteile, Briefe (...).“

Die Spießroutenläufe zu Behörden sowie zu anwaltlichen und ärztlichen Fachkräften werden von Maryam nicht so quälend empfunden wie das Warten an sich. Sie betont in unseren Gesprächen, dass sie eine außerordentliche Genugtuung fühlt, losgelöst von der patriarchalen Enge ihrer Heimat die Freiheit zu haben, sich zu bilden. Mit acht Jahren flüchtete sie mit ihrer Familie aus Afghanistan in den Iran. Maryam heiratete mit neunzehn Jahren. Damals blieb ihr nichts anderes übrig. Aus wirtschaftlichen Gründen musste sie bald das Elternhaus verlassen und ihrem Ehemann nach Teheran folgen. Andere Afghaninnen in ihrem Alter hatten laut Maryam außerdem bereits Kinder.

„Ich hatte wirklich Glück, denn meine Eltern ließen mich so lange wie möglich bei sich wohnen. Die Diskussion ging über ein Jahr lang, schließlich musste ich heiraten."

Ihr Mann sei anfangs wenigstens freundlich gewesen, wenngleich keine Liebe zwischen den beiden bestanden habe.

„Dann fing er mit den Drogen an und alles wurde schlimmer. Er verlor seinen Job auf dem Bau und fand daraufhin einfach keine Arbeit mehr. Mit seinen Kumpels fuhr er regelmäßig nach Afghanistan und brachte Drogen nach Teheran. Ich war einsam und konnte mit niemandem über meine Situation reden. Das war immer so. Hätte ich meinen Sohn nicht in die Schule schicken können, wäre ich verrückt geworden. [...] Ich lass mich ja von meinem Ex-Mann scheiden. Ich bereue es 
einfach nur, dass ich mit ihm nach Deutschland gekommen bin. Wir hätten uns wirklich so viel Leid ersparen können, wenn ich einfach nur mit meinem Sohn geflüchtet wäre. Jahrelang hat er mich behandelt wie seine Sklavin, (...) wie seinen Hund. Aber ich hatte wirklich Glück, denn geschlagen hat er mich nicht so oft. Er hat mich einfach immer nur runtergemacht und beschimpft. Ich hatte mehrere Male meine Sachen gepackt und wollte alleine mit meinem Sohn los, aber mir fehlte der Mut."

Sechzehn Jahre lang lebte Familie Y., bis kurz vor der Flucht, im Süden Teherans. Mehrere Male erzählt sie mir, wie verschachtelt und knifflig die Entscheidung war, mit ihrem ungeliebten Ehemann herzukommen. Schließlich mussten die Gefahren in Betracht gezogen werden, die für eine Mutter auf dem Fluchtweg lauerten. Zwischendurch bittet sie mich die Aufnahme zu stoppen. Ich teile ihr mit, dass dies kein Problem darstellt und frage nach ihrer Zeit in Teheran. In dieser Zeit beschäftigt sie sich intensiv mit ihren Studien an der Universität in Teheran. Sie schafft es unter widrigsten Umständen, die Gebühren für die Eignungstests aufzubringen, diese zu bestehen und anschließend an unterschiedlichen Universitätsveranstaltungen teilzunehmen. Sie bedauert allerdings sehr, dass sie keine der vielen Disziplinen, die sie begonnen hat, zu Ende studieren konnte. Sie studiert mehrere Semester Literaturwissenschaften und Medizin und findet durch die Teilnahme an den Veranstaltungen sozialen Anschluss. Für eine afghanische Frau existieren aber zu viele Hürden auf dem Weg zu ihrem akademischen Abschluss, als dass sie ernsthaft daran glauben könnte, Ärztin zu werden. Der freie Zugang zu Bildung bleibt ihr meistens verwehrt oder wird durch Bürokratie und übermäßige Gebühren behindert - nicht bloß, weil sie seit ihrer Kindheit als Angehörige der afghanischen Minderheit im Iran lebt, sondern angesichts ihrer festgeschriebenen Rolle als (Haus-)Frau. Sie berichtet, dass sie die Wartezeiten hier in der Bundesrepublik liebend gerne mit Deutschlektionen füllen würde, doch sie kann schlichtweg nicht. Durch die Ablehnung des Asylantrags gerät sie in eine Krise, die sie vollständig aus der Bahn wirft. Sie schläft und isst kaum, ist entsprechend mager und farblos. Sie benötigt zudem ein psychiatrisches Attest, um ihren Aufenthalt zu sichern. Wie etliche andere Asylbewerber*innen befindet sie sich in einer beunruhigenden Situation: Sie muss psychisch krank sein, um einen dauerhaften Aufenthalt in Deutschland zu erwirken. Maryams anwaltliche Fachkraft setzt genau auf diese Option. Seit dem Inkrafttreten des Asylpakets II aus dem Jahr 2016 sollen medizinische Abschiebehindernisse nur noch dann akzeptiert werden, wenn es sich laut Aufenthaltsgesetz $\S 60$ a (siehe Tabelle Nr. 1) um „lebensbedrohliche und schwerwiegende" Erkrankungen handelt (siehe Abschn. 3.2). Es muss hierbei individuell 
und konkret eine erhebliche Gefahr für Leib und Leben ersichtlich sein. Maryams Fall wird fortan am Verwaltungsgericht ${ }^{1}$ verhandelt, da das BAMF nicht mehr für abgelehnte Asylbewerber*innen zuständig ist. Das allein lässt sie bereits verzweifeln. Sie ist ausgesprochen aufgeregt, vor einem deutschen Gericht ihre Lebens- und Fluchtgeschichte zu erzählen, wie ich von ihr beim nächsten Treffen am Landesamt erfahre. Beim BAMF konnte sie nicht all ihre Fluchtgründe darlegen, weil sie zum damaligen Zeitpunkt noch ihren Mann schützen musste. Die ausgeklammerten, jedoch aufenthaltsrechtlich relevanten Aspekte ihrer Fluchtgeschichte - geschlechtliche Diskriminierung, häusliche Gewalterfahrungen und vor allem die Unmöglichkeit, ihr Recht von der iranischen Judikative einzufordern - sind mit Blick auf Maryams emotionale Verfassung nicht zu unterschätzen. Ihre Rechtsvertretung teilt ihr indes mit, dass sie von einer drei- bis vierjährigen Wartezeit ausgehen sollte. Maryam und ich sitzen auf den unbequemen grauen Stühlen des Landesamts und warten während unserer Unterhaltung auf ihren Termin. Sie trägt dunkle Kleidung und ein Kopftuch. Ihre vorderen Haarsträhnen ragen aus dem Schleier heraus und bilden eine Welle mit dem Schwerpunkt auf der linken Seite des Kopfes. Die restlichen Haarpartien sind mit Klammern unterhalb des Schleiers befestigt, so dass kein weiteres Haar - abgesehen von der Welle - zu sehen ist. Sie ist dünn und hat lange Arme und Beine. Ihre großen braunen Augen mit den dunklen Ringen unter ihnen fallen gleich auf und prägen, neben der länglich-spitzen Nase, ihr Gesicht. Sie sortiert gewissenhaft die vielen Briefe aus ihrer Tüte und kontrolliert immer wieder ihr Mobiltelefon, obwohl es nicht klingelt. Das endlose Warten auf die entscheidende Verhandlung als abgelehnte Geflüchtete wird zu einer Zerreißprobe für Maryam. Der Zugang zu sozialer Teilhabe wird für diese Personengruppe durch Verschärfungen der Asylgesetze erschwert, während sich die Lage in Afghanistan zunehmend verschlimmert (siehe Abschn. 3). Aufgrund der Residenzpflicht ${ }^{2}$ darf Maryam weiterhin nicht ihre einzige Freundin in Hamburg besuchen.

\footnotetext{
${ }^{1}$ Die sich häufenden Einsprüche gegen die vom BAMF beschiedenen Ablehnungen überfordern das Berliner Verwaltungsgericht maßlos, obwohl es im Jahr 2017 personell von 93 auf 113 Richter*innen aufgestockt wurde. Mehrere Jahre dürfte laut den anwaltlichen Fachkräften meiner Kontakte die Abarbeitung der Widerspruchsverfahren dauern.

${ }^{2}$ Asylbewerber*innen und geduldete Ausländer*innen unterliegen einer asyl- und aufenthaltsrechtlichen Pflicht, sich nur in einem bestimmten Bezirk aufzuhalten. Offiziell erweiterte Berlin als erstes Bundesland die Beschränkungen von Aufenthaltsgestattungen. In der Praxis hat diese Aufhebung jedoch keinerlei Auswirkungen. Während des Feldaufenthaltes gibt es etliche Schwierigkeiten aufgrund dieser Residenzpflicht.
} 
„Jetzt muss ich ein paar Jahre warten, bis mein Aufenthalt verhandelt wird. Diese Ungewissheit ${ }^{3}$ macht mich verrückt. Was soll ich sagen? Ich fühle, dass ich gescheitert bin im Leben. [...] Ich muss meinem Sohn in dieser schweren Zeit seines Lebens Mut machen. Er hat schon genug mitgemacht. Wissen Sie, als mich mein Ex-Mann das letzte Mal im Heim so heftig schlug, rief mein Sohn die Polizei. Er ist mein Retter. Er ist wahrlich mein Retter, mein lieber Sohn. Jetzt ist er auch noch vaterlos. [...]“

Ihr Sohn begreift, dass Gewalt im Ankunftskontext polizeilich geahndet wird. Als Maryams Mann wiederholt zuschlägt, benachrichtigt ihr Kind die Sicherheitskräfte der Unterkunft, die ihrerseits umgehend die Polizei verständigen. Maryams Mann wird an diesem Abend verhaftet und von ihr getrennt. Seitdem hat sie ihn nur einmal ganz kurz mit anwaltlicher Vertretung getroffen. Nach der Verhaftung ihres Mannes in der Berliner Unterkunft zeigen ihr Sozialarbeiter*innen in diversen Beratungsstellen Wege auf, sich unkompliziert scheiden zu lassen. Nach islamischer Praxis muss dazu jedoch die Billigung derjenigen Moschee vorliegen, welche die Eheschließung vollzogen hat. Bis der Papierkram erledigt ist, verstreichen viele Monate.

„Manchmal habe ich morgens Atemnot und Panik. Die Angst, nach Afghanistan abgeschoben zu werden, obwohl wir im Grunde noch nie da waren, bringt mich um. Manchmal ist es sehr intensiv, dann denk ich (...) ach, dann wäre alles vorbei. Können Sie sich vorstellen, was ich, (...) ich meine, was soll ich denn mit meinem Sohn gemeinsam in Afghanistan machen? Entweder ich schick meinen Sohn gleich in die Koranschule, zu den Taliban, oder (...) Der Iran war schon so schlimm, was wäre dann Afghanistan für uns?"

Beim diesem Thema zeigt Maryam starke emotionale Reaktionen. Ihre Stimme wird lauter, während sie wuchtig den Arm und den Zeigefinger ausstreckt und die erheblichen Diskriminierungen gegen die afghanische Minderheit beklagt. Mit einem Schlag spüre ich kraftvolle Wut in dieser Person. Ihre Mimik verändert sich plötzlich, ihre Augenbrauen senken sich, in ihren Augen sammeln sich Tränen, die ihr die Wangen herunterrollen, dann beruhigt sie sich wieder. Sie beugt und krümmt sich buchstäblich wieder in die vorherige, unterwürfige Position. Maryam reibt sich die Schläfen und atmet regelmäßig im Takt ein und aus. Sie fängt sich rasch, ahmt ein Lächeln nach, indem sie das Kinn durch eine stramme Bewegung nach oben schnellen lässt und ihre Lippen aneinanderpresst. Während sie beginnt, etwas in ihrer Handtasche zu suchen, schaue ich weg und schweige. Sie stellt wiederholt fest,

بى تكليفى 3 
dass sie doch dankbar zu sein hat, anstatt sich unablässig zu beschweren, während sie weiterhin tapfer und verkrampft lächelt. Diese Art von schlagartiger Veränderung empfinde ich als typisch für Maryams Art der emotionalen Ausdrucksweise. Häufig wechselt sie das Thema und spricht vom Deutschlernen.

„Das ist das Schöne am Deutschlernen - da vergesse ich immer all diese Sorgen. Es ist das erste Mal in meinem Leben, dass ich selbstbestimmt lernen kann, Bibliotheken und Museen besuchen darf, ohne einen Mann, der dir alles sagt, aber irgendwie (...). Ach, ich kann mich nicht freuen. Auch wenn mich alle trösten und behaupten, dass eine Abschiebung echt selten erfolgt, und das mag ja auch stimmen, aber (...). Wie soll ich das denn alles hier genießen? Sie kennen doch die Afghanen! Alle warten jetzt nur darauf, mich zu zerstören.“

Maryams Unterkunft ist nicht weit von der Innenstadt entfernt. Sie lebt seit einiger Zeit alleine mit ihrem zwölfjährigen Sohn in einem „Camp“, das etwas mehr Komfort bietet als die überfüllten Massenunterkünfte zuvor. Doch der Lärm und die vielen unüberhörbaren, heftigen Auseinandersetzungen, die beinahe täglich ausufern, bilden weiterhin einen wesentlichen Bestandteil ihrer Lebenswelt. Polizeibeamt*innen erscheinen anfangs so gut wie jeden Tag in den Massencamps, weil Handgreiflichkeiten unter den Geflüchteten eine banale Normalität darstellen. Die nicht wegzudenkende Anwesenheit von Sicherheitskräften und Polizei und die mit ihnen einhergehenden Ängste und Erwartungen in den Gebäuden der Institution Asyl stellen somit ein weiteres Merkmal derselben dar. Diese Eigenschaft gibt unmissverständlich zu verstehen, dass die Betroffenen mit Gefahr, Unberechenbarkeit und Bedrohung rechnen müssen, und begünstigt eine spezifische Grundstimmung, die typisch ist für die Institution Asyl.

„Zumindest kann ich jetzt zusammen mit meinem Sohn leben. Er kann jetzt auch Gott sei Dank regelmäßig zur Schule. Von meinem Stress wegen der Verhandlung kriegt er nicht viel mit.“

Gemeint ist der Termin am Verwaltungsgericht, an dem ihr Widerspruch gegen den abgelehnten Asylbescheid verhandelt wird. Dann lässt sie erkennen, weshalb sie jenseits der offensichtlichen Probleme der Institution Asyl beunruhigt ist. Beim nächsten Treffen, diesmal wieder am Landesamt, berichtet sie von ihren Sorgen bezüglich ihrer Verwandtschaft.

„Mittlerweile sind viele aus unserer Großfamilie auch in Berlin. Es ist wirklich fürchterlich. Jetzt bin ich hier in einem entwickelten Land und habe genauso Angst, von ihnen verurteilt zu werden, wie in der Heimat. Und es ist völlig egal, ob man als Frau ein Kopftuch trägt oder nicht (...).“ 
Sie hält sich mit ihren Fingern die Stirn fest, wobei jede Fingerkuppe resolut einen bestimmten Punkt massiert. Dabei atmet sie schwer und unterdrückt ihre Tränen. Ich verstehe nicht, was sie genau mit dem Tragen des Kopftuchs meint und frage nach.

„Meine Cousine zum Beispiel. Sie trägt ihr Haar ganz offen, genau wie die Deutschen. Aber wenn sie mit ihrem Ehemann rausgeht und nur einziges Mal einen fremden Mann ohne Erlaubnis anguckt, wird sie mit einer Ohrfeige bestraft.“

Sie greift ihr Mobiltelefon aus ihrer Handtasche und sucht nervös ein paar Fotos aus. Sie reicht mir das Handy. Währenddessen ändert sich ihre Gesamterscheinung. Sie verlangsamt die Geschwindigkeit ihrer Bewegungen, wischt sich in aller Seelenruhe mit einem Taschentuch die Tränen weg und setzt sich kerzengerade hin. Während sie ihre Souveränität demonstriert, schaut sie mich erwartungsvoll an. Auf dem Foto sind die Angehörigen zu sehen, die gerade freudig am Brandenburger Tor posieren. Nichts darauf erweckt den Eindruck, es könnte sich um eine Familie handeln, deren Frauen verprügelt werden, wenn sie außerhalb der eigenen vier Wände fremde Männer anschauen, ganz zu schweigen von anderen alltäglichen sozialen Interaktionen. Die eigentliche Absicht beim Verzicht auf das Kopftuch sei, so erklärt sie mir, im Ankunftskontext nicht negativ aufzufallen.

\begin{abstract}
„Andere 4 sind immer wieder aufgefallen, weil sie sich in der Öffentlichkeit stritten oder schlugen. Sie haben jetzt Probleme mit der Polizei. Die Frauen von denen, die jetzt Probleme haben, wollen ihre Haare offen tragen, dürfen es aber nicht. Afghanische Familien haben richtige Probleme damit. Meine Großfamilie ist da ganz anders. Wir wollen keine Probleme mit der Polizei. [...] Nach außen hin geben sie sich ganz westlich, um niemals in Schwierigkeiten mit den Behörden zu geraten; nur Theater! Keine Rede von Koran oder Kopftuch, gar nichts. Aber daheim herrscht die reinste Diktatur. Frauen werden für jede Kleinigkeit gemaßregelt und für jeden eingebildeten Blick auf andere Männer gibt es Prügel.“
\end{abstract}

Die Anpassungsstrategie der Großfamilie Maryams in Bezug auf den Umgang mit Frauen, insbesondere das Weglassen des Tschadors, begegnet mir gelegentlich auch bei anderen Kontakten. Mit steigendem Druck der Institution Asyl kommt es zu solchen Adaptionsstrategien der Mimikry (siehe Abschn. 4.5).

Bei einem weiteren Treffen sitzen Maryam und ich wieder im Landesamt und warten, wohl wissend, dass der heutige Termin nicht stattfinden wird.

${ }^{4}$ Gemeint sind andere afghanische Asylbewerber*innen in Berlin. 
Zahlreiche Geflüchtete laufen währenddessen in Gruppen zu den verschiedenen Häusern auf dem Gelände. Unter ihnen verteilen die Zeugen Jehovas und andere christliche Sekten ihre jeweiligen Heilsschriften, darunter vor allem aus den USA stammende evangelikale Christen. Sie versprechen Asylbewerber*innen nicht selten einen festen Aufenthalt, wenn sie konvertieren. Dies sorgt bei zahllosen Anhörungen und Asylanträgen für Verwirrung und Frust auf allen Seiten. Beim Vorbeigehen werde ich schließlich auch gefragt: „Arabic? Farsi?“ Ich nicke einfach bei „Farsi“ und bekomme eine Bibel in die Hand gedrückt. Wir setzen uns schließlich raus auf die Bänke bei den wenigen Grünflächen des Landesamts und warten mit einer Bibel und einem Beutel frischer Datteln in den Händen darauf, dass Maryams Sohn von einer Sport- und Spielgruppe zurückkehrt.

„Ich bin so dankbar, dass ich auf die Uni gehen konnte in Teheran. Zwar wusste ich von Beginn an, dass ein Abschluss für eine Afghanin höchst unwahrscheinlich ist, aber das war mir natürlich lieber, als den ganzen Tag daheim zu sein. Während der Schulzeiten meines Kindes war ich immer an der Universität. Mein Mann war zwar auch unterwegs, aber ich wollte unbedingt studieren. Lernen machte mir immer schon Spaß; und ich hatte erstmals Freundinnen (...), die wussten gar nicht, dass ich eine Afghanin bin, weil mein Farsi so akzentfrei ist. Das war herrlich. Mein Gott. (...) Damals war ich überzeugt, dass Frieden und Gerechtigkeit ausschließlich in einer islamisch regierten Gesellschaft möglich seien. Jetzt aber weiß ich, dass das falsch war. Früher hätte ich auch niemals so viel Haare gezeigt. Doch jetzt (...). Ach, alles ist anders. Jetzt habe ich mich so sehr an dieses Ding gewöhnt (...). Naja, ich dachte auch schon, den Schleier einfach beiseite zu lassen. Aber dann wäre ich (...) die Hure, die sich von ihrem Mann getrennt hat und gleich all ihren Glauben, ihre Werte und ihre Kultur in die Tonne schmeißt, um den westlichen Lebensstil zu zelebrieren. Sie können sich ja nicht vorstellen, wie die Afghanen so sind. Ich habe es am Anfang in Deutschland mitbekommen, als ich noch mit meinem Mann zusammen war. Sobald eine Frau den Schleier nicht mehr tragen oder die Beziehung beenden wollte, sprach sich das rum. Innerhalb eines Tages wusste jeder im Heim Bescheid. Ach, Heim, was sag ich da? Ganz Berlin weiß gleich Bescheid. Ich bin bald offiziell getrennt. Vielleicht werde ich dann mehr Haare zeigen. Eigentlich geht es mir auch gar nicht um die Haare. Sie können sich das einfach nicht vorstellen. Meine Kultur bedeutet mir auch was, aber (...). Ich dachte, ich werde es packen (...), weil ich es ja gewohnt bin. Als ich das erste Mal in Deutschland realisierte, dass mein Kopftuch wegkann, fühlte ich etwas Großartiges ${ }^{5}$. Ich könnte auch Teil einer modernen Gesellschaft sein, aber seitdem ging es nur noch bergab, ganz plötzlich. Die Trennung, die Ablehnung, die Gerichtstermine, die Angst, abgeschoben zu werden, und all dies.

\footnotetext{
außerordentlich, außergewöhnlich, grandios, exorbitant, besonders.
} 
Maryam erlebt den Druck innerhalb der Institution Asyl als niederschmetternd. In ihren Interaktionen verhält sie sich unterwürfig und betont ihre Sittsamkeit. Sie senkt förmlich den Kopf, wenn ich mit ihr spreche, und hebt ihn wieder an, wenn sie antwortet. Dabei legt sie die eine Hand auf die andere und streichelt diese behutsam. Ich spüre eine religiös motivierte Angst seitens Maryam, unsere Interaktionen könnten als unmoralisch verstanden werden, was sie auf meine Frage hin bestätigt. Es sei ihr eigentlich untersagt, mit fremden Männern zu reden. Sie möchte länger als vorgeschrieben warten, bis sie sich offiziell als ledig bezeichnet. Die Quellen ihrer moralischen Regeln kann sie nicht genau darlegen. Nachdem der Termin nicht stattfindet, verabschiedet sie sich und kehrt mit ihrem Kind ins Camp zurück.

Warten bildet einen Eckpfeiler in der Lebenswelt Maryams, den ich in unserer gemeinsamen Zeit sehr intensiv miterlebe. Goffman greift den Gesichtspunkt des Wartens in Asyle auf und betont in seiner Analyse, dass die Wartezeit in der totalen Institution eine ,verlorene, vergeudete und nicht gelebte Zeit" sei, die ,irgendwie ,abgesessen “ oder ,durchgestanden“ oder , hinter sich gebracht" werden“ müsse (1973: 71). Dabei werde die Wartezeit bewusst ausgeklammert, während sich der Untergebrachte in seinen Gedanken ,dauernd mit ihr in einem Maße, wie dies draußen nicht üblich ist", beschäftige; Goffman verwendet hier den Begriff „Folter“ (ebd.: 72). Dieser Aspekt bildet in seiner Untersuchung ein Merkmal, mit dem alle Insassen gleichermaßen konfrontiert werden. Das ständige Warten innerhalb der totalen Institution Asyl wird ebenso von Asylbewerber*innen als Form der Schikane und Machtausübung empfunden. Vor diesem Hintergrund und angesichts der rigorosen Residenzpflicht ist Maryam unentwegt mit ihrem eigenen Schicksal, das sie als Scheitern deutet, konfrontiert. Durch das Warten in den Räumlichkeiten der Institution Asyl wird der wartenden Person das „eigene Missgeschick“ (Goffman 1973: 70) vor Augen geführt. In Goffmans Studie handelt es sich dabei um die Tatsache, sich in der stationären Psychiatrie zu befinden. Der Begriff „Missgeschick“ impliziert die eigene Verantwortung und weist gleichermaßen darauf hin, dass es sich um etwas handelt, dessen sich die betreffende Person zu schämen hat. Dies führt dazu, dass Insassen eine Neigung entwickeln, sich übermäßig mit sich selbst zu beschäftigen, um gewissermaßen eine Rechtfertigung für ihren „,niedrigen Status“ (ebd.) zu finden, etwa in Form einer speziellen Wehklage, eines traurigen Berichts oder einer Apologie (ebd.).

„Die Insassen müssen dazu gebracht werden, sich selbst in der Weise zu steuern, dass sie leicht $\mathrm{zu}$ verwalten sind. Und um dies zu erreichen, wird sowohl das erwünschte wie das unerwünschte Betragen als etwas definiert, das dem 
persönlichen Willen und dem Charakter des einzelnen Insassen selbst entspringt und wofür er selbst verantwortlich ist" (ebd.: 89).

Analog besteht das „Missgeschick“ im untersuchten Feld darin, ein „abgelehnter Flüchtling" in der Institution Asyl zu sein. Im Vergleich zu Goffmans totaler Institution addiert sich neben der zeitlichen Komponente des Wartens die knapp bemessene räumliche Dimension (vgl. dazu die rechtlichen Bestimmungen in Abschn. 3.2). Bevorzugte Gruppen von Asylbewerber*innen erhalten mehr Raum und Komfort. Entscheidend sind hierbei die geringen Bleibeperspektiven der benachteiligten Gruppen. Afghanische Geflüchtete etwa betonen die Überlegenheit von Narrativen der eigenen Referenzgruppe. Daraus entwickeln sich angesichts der Gefährdung des Status ihrer Referenzgruppe neue Narrative, die es erlauben, „konkurrierende“ Gruppen abzuwerten. Dadurch gelingt es, eigenen politischen, wirtschaftlichen, sozialen und humanitären Problemen mehr Gewicht zu verleihen und diese anschließend zu exponieren. ${ }^{6}$ Das „Missgeschick“ wird damit zusätzlich auf einer abstrakteren Ebene gerechtfertigt. Bei der Überbietung des Leids anderer Menschen während des Asylprozesses zeichnet sich ein Konkurrenzkampf um Anerkennung seitens der Einwanderungsgesellschaft ab. Angesichts der Bevorzugung anderer Gruppen von Asylbewerber*innen und der medialen Aufwiegelung ${ }^{7}$ gegen Afghan*innen bemerke ich bei Maryam, wie bei anderen Kontakten einen sehr starken Drang, ihre Flucht nach Deutschland zu rechtfertigen. Hinzu kommen tägliche Begegnungen, bei denen im Grunde auf Knopfdruck $^{8}$ die Fluchtgeschichte erzählt werden soll, am besten in einem Satz. Es ist bemerkenswert, dass entsprechende Fragen äußerst unvermittelt und oft ohne einen Ansatz von Empathie gestellt werden ${ }^{9}$ - auch diese Taktlosigkeit erscheint als eine Facette des totalitären Merkmals der Fragehoheit.

\footnotetext{
${ }^{6}$ Vgl. dazu den Ansatz der sozialen Dominanztheorie von Sidanius/Pratto 1999 und Tajfel 1978; 1981; Tajfel et al. 1971; Tajfel/Turner 1986.

${ }^{7}$ Meine Gesprächspartner*innen sind über soziale Medien bestens über die deutsche Innenpolitik informiert und reagieren zeitnah auf Auftritte und Kundgebungen deutscher Politiker*innen im Zusammenhang mit afghanischen Geflüchteten, die zumeist als aufhetzend und populistisch begriffen werden.

${ }^{8}$ Etwa bei Standardfragen, die bei ärztlichen Fachkräften vor der eigentlichen Behandlung erhoben werden.

${ }^{9}$ Dies erfolgt oft sowohl während der BAMF-Anhörungen als auch während gewöhnlicher Sitzungen bei Ärzt*innen.
} 
Infolgedessen entwickeln Geflüchtete entsprechende Narrative, um das eigene Scheitern (,Missgeschick“) über die Rechtfertigung hinaus mit Bedeutung aufzuladen und auf diese Art mit anerkannten Geflüchteten ,konkurrenzfähig“ zu sein. Das Adjektiv, das im Zusammenhang mit dem Gefühl des Scheiterns (احساس شكست) hauptsächlich verwendet wird (بدبخت: ,badbakht“), ist bereits im ersten Fallbeispiel erläutert worden und formt das Narrativ des „Missgeschicks“ bei Maryam maßgeblich mit. Viele Afghan*innen spüren die soziale Benachteiligung in Deutschland allzu deutlich, selbst bei bewilligtem Asylantrag. Dieser Umstand wird mir gegenüber unmissverständlich und unentwegt kommuniziert. Rashid A. (38) und seine Ehefrau Nilufer (29) etwa, zwei Geflüchtete aus Ghazni in Zentralafghanistan, die ihre asylrechtliche Anerkennung erhalten haben, berichten mir Anfang 2017 am Eingang des medizinischen Zentrums des Landesamts:

\begin{abstract}
„Das Leben und alles (...) ist nicht so einfach mit einem ausländischen Namen und so (...). Wir haben zwar den Aufenthaltstitel erhalten, aber ich finde weder eine Wohnung noch einen Job. Wir müssen uns für alles bewerben. Ich habe das bestimmt schon tausend Mal gemacht. Es klappt einfach nicht. Wir müssen dennoch mit den anderen ${ }^{10}$ im Camp leben. Das ist kaum zu ertragen für uns.“
\end{abstract}

Das Gefühl der systematischen Benachteiligung angesichts eines „Missgeschicks", dessen man sich schämen und für das man sich rechtfertigen sollte, ist maßgeblich für die Lebenswelt Maryams. Der Kern ihrer Lebenswelt ist untrennbar verbunden mit dem Prinzip der ethnischen Hierarchisierung, das bereits im ersten Fallbeispiel thematisiert wurde. Die Gespräche mit Maryam handeln sehr häufig davon. Syrische Asylbewerber*innen warten meist nur wenige Monate, bis sie eine dauerhafte Unterkunft erhalten, während sich die meisten afghanischen Asylbewerber*innen nach über zwei Jahren noch in Erstaufnahmeeinrichtungen aufhalten müssen. Die ungleiche Dauer der Asylverfahren ${ }^{11}$ und die unverständliche Verteilung der Asylbewerber*innen auf Sprachkurse - zwei wesentliche Säulen des Ankunftskontextes - kommen hinzu. Die Ungewissheit als charakteristischstes Merkmal des Wartens wirkt in diese Dynamik hinein. Bei Asylbewerber*innen aus benachteiligten Gruppen dauert es teils Jahre, bis sie regelmäßig eine Bildungseinrichtung, Schule oder Ähnliches besuchen dürfen. Der Ankunftskontext bereitet Maryam dauerhaft

\footnotetext{
${ }^{10}$ Gemeint sind andere Asylbewerber*innen, die keine Aufenthaltsbestätigung erhalten haben.

${ }^{11}$ Siehe Abbildung 4.
} 
Stress und bietet keine Sicherheit. Die Abschiebepraxis sieht vor, dass afghanische Asylbewerber*innen nach Afghanistan abgeschoben werden, selbst wenn sie als Geflüchtete lange Zeit in anderen Staaten lebten (meist im Iran oder in der Türkei). Vorstellungen darüber, wie ein Leben mit ihrem Sohn in Afghanistan aussehen würde, tragen zu Maryams Unwohlsein bei. Für die Intervention ihres Jungen ist sie ungemein dankbar. Die Verantwortung, die Beziehung zu beenden, bürdet sie damit unbewusst ihrem Sohn auf. Allerdings ist ihr Verantwortungsbereich hinterher spürbar gewachsen. Denn neben der drückenden Last des Lebens im Flüchtlingsheim als abgelehnte Asylbewerberin muss sie nun eine Trennung mit all ihren Implikationen und bürokratischen Verwicklungen durchstehen. Über längere Zeit hinweg hat sie angesichts dessen das Gefühl der Ohnmacht. Sie sieht sich der Aufgabe nicht gewachsen, Einfluss auf ihren unerträglichen Zustand auszuüben. Auf die Einschätzung (Emotionsbaustein I) ihrer Situation und die körperlichen Veränderungen folgen unbestimmte, erdrückende Gefühle. Damit beginnt die Transformation (Katz I) ihres Körpers als Träger und gleichsam als Mittel emotionaler Ausdrucksweisen, was durch die Resonanz der sozialen Interaktionen (Katz II) verstärkt wird. Aus den zunächst unbestimmten Affekten und Gefühlen der Verunsicherung und Handlungsunfähigkeit wächst mit der Zeit eine dominierende Emotionsfärbung heran, die sich aus unterdrückter Wut, Unterlegenheit und Verunsicherung konstituiert. Neben den typischen Depressionssymptomen - Gewichtsabnahme, schwerwiegende Schlafstörungen, Vergesslichkeit, Antriebslosigkeit - bilden wiederkehrende Panik- und Wutattacken die deutlichsten Anzeichen ihrer spezifischen sinnlichen Metamorphose. Maryam berichtet von kontinuierlich auftretenden „Stresszuständen" 12 und nimmt jedes Mal ein halbes Dutzend Pillen, die sie bei diesen Anfällen nehmen soll, aus ihrer Handtasche, darunter die populären Benzodiazepine. Eine andere Facette ihrer sinnlichen Metamorphose besteht aus der Trennung von ihrem Ehemann, denn die körperlichen und verbalen Angriffe bleiben künftig aus. Infolgedessen erhalten Gefühle Raum, die lange unterdrückt werden mussten. Die Dimension der Leiblichkeit im Prinzip der sinnlichen Metamorphose spielt demnach im vorliegenden Fall eine besondere Rolle. Besonders auffallend sind in diesem Zusammenhang ihre plötzlich auftretenden Wutattacken („Stresszustände“). Diese werden teils durch Erinnerungen an die Heimat ausgelöst. Dadurch wird der Emotion Wut sozialer Sinn verliehen, indem sie Maryams damalige Situation neu bewertet und durch die Aufführung ihrer spezifischen Narration (Katz III) die Wiederherstellung ihrer verletzten Würde zu gewährleisten sucht. Maryam ist zwischen Herkunfts- und

حالت هاى استرس 12 
Ankunftskontext sowie ihren entsprechenden Gefühlen hin- und hergerissen. Die Inszenierung dieser zwei Seiten ihrer Gefühle im Dritten Raum ist das soziale Drama, das in unseren Interaktionen herangebildet wird und ihre Lebenswelt prägt.

Bevor die spezielle Narration Maryams untersucht wird, soll eine methodologische Schwierigkeit meiner Forschung angesprochen werden. Das tägliche Zusammensein mit Geflüchteten hat das Potenzial, bei zu unterschiedlichen Lebensauffassungen unangenehme Situationen hervorzurufen, so beispielsweise in Bezug auf soziale Verhaltensregeln in Interaktionen mit afghanischen Frauen. Ein Zusammentreffen mit einer afghanischen Asylbewerberin beginnt damit, dass ich innerhalb eines Augenblicks entscheiden muss, ob ich zur Begrüßung die Hand ausstrecke oder nicht. Meist unterlasse ich den Handschlag, um meine Seriosität als Dolmetscher nicht zu riskieren. Aus der spontanen Unterdrückung des Handschlags erwächst jedoch ein Gefühl des Unmuts in mir, das seine Ursache darin hat, dass weibliche Geflüchtete bisweilen das westliche Ritual des Händeschüttelns bewusst ausüben und sichtlich Gefallen daran finden. Gebe ich ihr also nicht die Hand, bestätige ich althergebrachte Denkweisen, die meiner Meinung nach einer Emanzipation entgegenstehen. Solche Spannungen und Dilemmata begleiten mich unablässig während des Feldaufenthalts. Hier spielen sicherlich auch Aspekte meiner Biografie eine Rolle. Ich komme aus einer Familie, die unter widrigsten Umständen geflüchtet ist, um Freiheit und geschlechtliche Gleichstellung (er-)leben zu können, ohne sich vor einem rigiden Gottesstaat reglementieren lassen $\mathrm{zu}$ müssen. Für patriarchale Praktiken zur systematischen Benachteiligung und Unterdrückung von Minderheiten bin ich vor diesem Hintergrund besonders sensibilisiert. Um den Aspekt der Selbstreflexivität transparenter zu machen, sei ein weiteres Beispiel erwähnt: Wenn ein Mann mitten im Gesprächstermin seine Ehefrau anherrscht, den Mund zu halten, während er sämtliche Angelegenheiten, selbst diejenigen seiner Gattin, an sich reißt, bin ich mitnichten neutral. Dessen bin ich mir bewusst, vor allem, um mich nicht unwillkürlich in die Dynamik sozialer Situationen zu ,,verstricken“ (Breidenstein et al. 2013: 39).

Die Dynamik im Feld bringt es mit sich, dass ich häufig in andere Rollen schlüpfen muss, um schlichtweg während des teilnehmenden Beobachtens nicht aufzufallen; wenn ich etwa als „Asylbewerber getarnt" ${ }^{\star 13}$ das Verhalten der Bediensteten und Asylbewerber*innen in den verschiedenen Bereichen der

\footnotetext{
${ }^{13}$ Zwecks meiner persönlichen Tarnung trage ich einfach eine abgetragene Sporthose im Feld und gehöre damit bereits zu ,den Anderen“.
} 
Institution Asyl beobachte. Das Bewusstmachen persönlicher Eigenheiten, die sich aus meiner Biografie ergeben (siehe weiter oben), entwickelt sich daher rasch zu einem „Ritual“, das ich immer vornehme, bevor ich das Feld betrete. Entsteht in der Folge während des Feldaufenthalts ein beklemmendes Gefühl, kann ich dieses schneller verstehen. In diesem Fall versuche ich nicht unberührt zu bleiben, sondern meinen Impuls weitestgehend bewusst wahrzunehmen, ohne ihn zu unterdrücken. Anschließend wird dies im Protokoll fixiert. Diese Vorgehensweise hilft, in Situationen wie der beschriebenen die Verhaltensweisen anderer Akteur*innen ebenso wie eigene Gefühlsregungen beobachten zu können, ohne mich in Wertungen zu verlieren. Doch dies ist nicht immer ohne Weiteres möglich, nicht zuletzt angesichts meiner Rolle als Hybrid im Feld. Die verschiedenen Rollen - als Sprach- und Kulturmittler, als ehrenamtlicher Helfer oder als Forscher - bringen je nach Kontext unterschiedliche, teils sich widersprechende Affekte und Ausdrucksweisen hervor. Ich stehe unentwegt und notgedrungen im Dazwischen. Aus diesem Grund kennzeichnet unter anderem ein Gefühl des „Ausgeliefert-Seins“ (Breidenstein et al. 2013: 40) meine Mehrfachrolle im Feld. Den Impuls, eine Gesprächsrunde schnellstmöglich zu verlassen, fasse ich primär als Quelle der Erkenntnis auf. Nach Episoden wie der beschriebenen im Gesprächstermin, fühle ich Frustration und Verwirrung. Erst nach einem langen und schwierigen Prozess vermochte ich einigermaßen das „Ausgeliefert-Sein“ und die daraus resultierenden Gefühle als epistemologische Fenster zu verstehen. Eigene Gefühle als erkenntnisleitend zu verstehen und einzusetzen ist sicherlich eine der größten Herausforderungen meiner Forschung. Ausschlaggebend ist, über das Sensorium $\mathrm{zu}$ verfügen, aufkeimende Emotionen, Projektionen und Übertragungsbilder als solche zu erkennen und in ihren situativen, biografischen und kulturellen Kontexten zu deuten (vgl. Bonz et al. 2017: 11 f.). Die Gegenübertragung wird hierbei als einziges Datum begriffen, das potenziell Informationen enthält, die man auf anderem Weg nicht erhält und die einzig aufgrund der im forschenden Subjekt empfundenen Störung festgehalten werden können (Devereux 1973: 308). Daher ist die Selbstreflexion im Zuge des zirkulären Forschungsprogramms von äußerster Relevanz, sofern man überhaupt bereit ist, solche Gegenübertragungen und die mit ihnen einhergehenden Emotionen und „Irritationen“ (Nadig 1986: 36) während des Feldaufenthalts wahrzunehmen (Matter 1978: 299). Denn gründliches Wahrnehmen führt zum Reflektieren und Erleben der emotionalen Mosaiken, mit denen man konfrontiert wird.

In diesem Fallbeispiel verläuft die Übertragung oder Ansteckung der Emotionen auf mich sehr ausgeprägt. Diese Ansteckung spielt eine nicht unerhebliche Rolle für eine ethnografische Forschung, zumal der Fokus der vorliegenden Studie auf Emotionen bzw. emotionale Ausdrucksweisen gerichtet ist. Darüber hinaus stellen, wie bereits betont, eigene Gefühle Daten im Sinne der Gegenübertragung 
dar, die als epistemologische Fenster $\mathrm{zu}$ verstehen und $\mathrm{zu}$ gebrauchen sind. Die Reflexion dieser Relation soll deshalb nicht fehlen. Ein Eintrag aus dem Feldtagebuch verdeutlicht den Grad der Ansteckung:

\begin{abstract}
„Maryams Nervosität ist anstrengend und strahlt auf mich ab. Ihre plötzlichen Wutanflüge dauern zwar nicht lange an, doch merke ich deutlich, wie sich ihre intensiven Emotionen auf mich übertragen. Im Vergleich $\mathrm{zu}$ anderen Kontakten, die naturgemäß ebenfalls verzweifelt und gestresst sind, ist die Abfärbung ihrer Empörung wuchtiger. Das Phänomen der emotionalen Ansteckung ist bei Maryam sehr präsent. Ich merke, wie ich in meinem Privatleben die Probleme Maryams gedanklich durchgehe, auch in meinen Träumen, und kurze Zeit darauf ihre, d. h. ,fremde Affekte' an mir identifiziere, die ich nicht so schnell loswerde. Nach einem intensiven Gespräch mit Maryam bin ich tagelang leicht erregbar und ungehalten. Ich spüre, dass ich so langsam an meine Grenzen komme in dieser Forschung. Die permanente Auseinandersetzung mit meinen Forschungssubjekten ,irritiert ${ }^{\star}$ mich gelinde gesagt. Zudem frage ich mich, ob und inwiefern diese unsagbaren Affekte adäquat in eine wissenschaftliche Studie übersetzt bzw. integriert werden können.“
\end{abstract}

Emotionale Ansteckung, „emotional contagion“, geschieht unbewusst und kann daher nicht gesteuert werden (Hatfield/Cacioppo/Rapson 1994: 5). Emotionale Ansteckung beruht auf der menschlichen Neigung, Gesichtsausdruck, Körperhaltung und Gestik unseres Gegenübers nachzuahmen. Bei Tieren ist die Tendenz der Nachahmung des Verhaltens von Artgenossen ebenfalls zu finden (vgl. Miller/Banks/Ogawa 1963). Laut Bargh, Chen und Burrows (1996: 232) spiegeln Menschen instinktiv und automatisch das emotionale Ausdrucksverhalten ihrer Interaktionspartner*innen. Ciompi spricht in diesem Zusammenhang sogar von einer „Versklavung“ (1997: 249), was dem erwähnten „Ausgeliefert-Sein“ (Breidenstein et al. 2013: 40) im Feld nahekommt. Das automatische Spiegeln der Emotionen vollzieht sich dabei auf der Grundlage einer Verknüpfung von Wahrnehmung und Verhalten. Der sogenannte PerceptionBehavior-Link zeigt, dass schon die bloße Wahrnehmung anderer Personen zur Annahme von deren Verhaltensweisen führt (Lohmann/Pyker/Zanger 2015: 66). Die subjektiv wahrgenommenen Verhaltensweisen aktivieren neuronale Schemata, die für das Ausführen des wahrgenommenen Verhaltens verantwortlich sind. Durch instinktives Nachahmen übernimmt man „,wie von selbst“ (Lakin et al. 2003: 145) die Emotionen des Gegenübers, um durch das Nachfühlen ein umfassenderes Verständnis seiner Wirklichkeit zu erlangen (Hatfield et al. 1994: 188). Dies erfordert eine spezifische Haltung der forschenden Person, die mit dem Aufsaugen und Nachahmen von Emotionen anderer einhergeht. Dieser Gesichtspunkt ist aus methodologischer Sicht relevant, da die ethnografisch-lebensweltliche Forschung das Mitteilen intimer Gefühle und Lebensgeschichten der untersuchten Menschen zu initiieren beabsichtigt. Emotionale Ansteckung funktioniert aber 
nur dann, wenn die empfangende Person sich auf irgendeiner Ebene mit den Emotionen der sendenden Person identifizieren kann, das heißt, es müssen bereits affektiv-kognitive Eigenstrukturen des empfangenden Individuums vorliegen, die ihrerseits eine emotionale Ansteckung ermöglichen (Lohmann et al. 2015: 46). Intensität und Authentizität nachgefühlter Emotionen hängen somit vom Ausmaß der affektiv-kognitiven Eigenstrukturen der empfangenden Person ab; fehlen sie, kann keine emotionale Ansteckung stattfinden (vgl. Ciompi 1997: 249 f.) Im Kontext der vorliegenden Studie bedeutet dies, dass die jeweilige Intensität der nachgefühlten Emotionen die Dimension der genannten Eigenstrukturen bei mir selbst erkennen lässt.

Die in diesem Fallbeispiel beschriebene Strategie der Großfamilie Maryams deute ich als Widerstandsform in der Ambivalenz des Dritten Raums. Damit werden die Ambivalenzen der verschiedenen kulturellen Gesinnungen sichtbar. Die Anpassungsstrategie der Mimikry besteht hier darin, dass weibliche Mitglieder der "Minderheit“ (Knecht) die weiblichen Mitglieder der "Mehrheit“ (Herr) nachahmen. Es wird ein Bild einer Identität reproduziert, das als Tarnung fungiert und nicht mit dem Original übereinstimmt. Das „Original“, also die tatsächliche in ihrem Lifestyle freie Frau, die mit ihrem Haar macht, was auch immer sie und einzig sie allein für richtig hält, wird durch die herausgebildete Ähnlichkeit aufgrund deren partiellen Charakters ambivalent. Die Familienmitglieder Maryams greifen die Symbole und Zeichen des „dominanten Diskurses" (Bhabha 2000: 131) auf, integrieren sie in ihr eigenes Symbol- und Zeichensystem und schaffen durch die prinzipielle Ambivalenz einen Diskurs, der bei performativer Wiederholung zur „Karikatur“ (Simson 2011: 75) geraten kann. Die aus dieser Mimikry hervorgehende „doppelte Perspektive“ (Bhabha 2000: 130) ist entscheidend: Einerseits eröffnet die täuschende Ähnlichkeit den unterdrückten Frauen neue Handlungsspielräume im eng geschnürten Ankunftskontext Berlin. Andererseits kann der dominierende Diskurs durch die performative Wiederholung der Ambivalenz kultureller Differenz angegriffen und destabilisiert werden (ebd.: 131). In dieser Konfiguration wird der dominierende Diskurs der Mehrheitsgesellschaft dadurch bedroht, indem er genötigt wird, seinen Text - in welcher Form auch immer, sprachlich, symbolisch, physisch usw. - ständig zu (re-)produzieren, um die eigene Macht und Autorität zu betonen und festigen. Damit kommt zum Ausdruck, dass der dominierende Diskurs doch nicht so stabil und monolithisch ist, wie es den Anschein hat. Die Schlagkraft der Mimikry setzt sich aus diesem Moment zusammen, der die koloniale Autorität verunsichert und aus Mimikry „Bedrohung und Ähnlichkeit in einem“ (ebd.: 127) macht. Die Repräsentation von Identität und Bedeutung wird bei diesem Übersetzungsakt metonymisch reartikuliert (ebd.: 133). Mithilfe der aus dieser 
Reartikulation hervorgehenden Entfremdung zwischen Signifikant und Signifikat wird eine neuartige (Be-)Deutung - Re-Signifikation - konstituiert (vgl. Abschn. 4.5). Das „Bedrohliche“ der Mimikry resultiert aus ihrer strategischen Produktion widersprüchlicher, phantastischer, diskriminatorischer „Identitätseffekte“ im Spiel eines Machtphänomens, das nicht dingfest zu machen ist (ebd.). Die spezifische Mimikry im vorliegenden Fall setzt präzise an einem prominenten Punkt der Differenz an - die Frauen der „Anderen“ tragen im Unterschied zur „eigenen Gruppe“ Kopftücher. Dieser Diskurs wird durch die Mimikry weder negiert noch bestätigt, sie legt vielmehr die innere Brüchigkeit des dominierenden Diskurses dar. In der Hybridität des Dritten Raums existieren schlichtweg keine distinkten Zuordnungen und Grenzen, kein widerspenstiger Gegendiskurs und keine devoten Unterordnungen (Struve 2013: 143). Das subversive Element der Mimikry besteht unter anderem in der Tatsache, dass sie eine Untergrabung des vorherrschenden Machtdiskurses ermöglicht. Imitation produziert im beschriebenen Kontext Subversion, etwa durch Widerstand im Inkognito-Zustand, bei dem nicht mehr deutlich zwischen Unterwerfung und Herrschaftsanspruch differenziert werden kann. „Die Freiheit, kein Kopftuch tragen zu müssen“, als wesentliche Eigenschaft der westlichen Kultur, wird in dieser Mimikry gewissermaßen zu einer Farce. Angesichts dieser Maßnahmen wird das Selbst „,beider Seiten“ gespalten. Der Kern beider Kulturen wird ausgehöhlt. Ihre in der Ambivalenz gefundene Differenz wird auf eine verstörende Art realisiert. Denn beim kolonialen Aufeinandertreffen der ,weißen Präsenz“ (Bhabha 2000: 133) mit ihrem „schwarzen Widerschein“ (ebd.) subordiniert sich der „Knecht“ zwar, doch erschüttert er durch Mimikry die Macht des „Herrn“. Durch diese Mimikry, die sich im Übrigen nicht einfach neutral ereignet, sondern mit Affekten der Verunsicherung und Verachtung im neuen Dritten Raum Deutschlands begleitet wird, deckt „,die Minderheit“ verborgene Ängste „der Mehrheit“ auf. Die Macht der westlichen Kultur wird, wenn man so will, verschoben und entfremdet. Die agency, die in dieser einzigartigen Hybridität ausgebildet wird, entfaltet sich nur in einem kleinen Spektrum und betont eher die performative Dimension der Mimikry. Im Dritten Raum können kulturelle Symbole neu verhandelt, mit neuen Bedeutungen versehen und damit neu gedeutet werden. Der „Dritte Raum der Äußerung zerstört den Spiegel der Repräsentation“ (Bhabha 1994: 38, zit. in Varela et al. 2015: 249), durch den Wissen als allgemeingültig verhandelt wird. Die Kopftuch-Mimikry ist nicht als dialektisches Spiel zwischen gegensätzlichen Polen - Orient vs. Okzident - zu verstehen, sondern als ein inkommensurabler „Zwischen-Ort“ (ebd.), der neue Positionen ermöglicht (agency) und die Grenzen des Diskurses umstürzlerisch herausfordert. Bhabhas Begriff der Mimikry ortet agency gerade dort, wo die westliche Kultur mitsamt ihrer Normen und Werte 
„angenommen“ wird (Castro Varela/Dhawan 2015: 230). Die durch das subversive Imitieren entwickelte Hybridität wirkt sogar gemeinschaftsbildend und stärkt die soziale Solidarität (Bhabha 2000: 297 f.) innerhalb Maryams Großfamilie. Somit trägt die Hybridisierung das Potenzial in sich, aufgrund der Unbestimmtheit für Panik innerhalb der Mehrheitsbevölkerung zu sorgen.

Bhabha bringt in diesem Zusammenhang ein Beispiel aus der indischen Kolonialzeit. Das „Gerücht“ sei ein ideales Verfahren gewesen, um sozialen Zusammenhalt bei den Kolonialisierten und zur selben Zeit Panik und Verwirrung bei den Kolonisatoren herbeizuführen. Kurz vor der Revolte von 1857 mussten die indischen Brote, „Chapatis“, von Dorf zu Dorf getragen werden, wobei die Übergabe der Chapatis mit dem Austausch von Geheimbotschaften assoziiert wurde (ebd.: 300). In diesem Prozess verselbstständigen sich Brote zu stillen, kryptischen und kraftvollen Zeichen, die die inferiorisierten Subjekte beschützen und Solidarität stiften.

„Die Indeterminiertheit des Gerüchts konstituiert seine Bedeutung als sozialer Diskurs. Seine intersubjektive kommunale Bindekraft liegt in seiner Äußerungsform. Die performative Macht seiner Zirkulation resultiert im ansteckenden Charakter seiner Verbreitung [...]“" (Bhabha 2000: 299).

Postkolonialer Widerstand bildet sich aus der Indeterminiertheit des Wesens eines Gerüchts, das als subversive Strategie sogar effektiver wird, weil Affekte und Panik heraufbeschworen werden (Struve 2013: 115). Die Bedeutung indigener Zeichen, hier des Kopftuchs, verschiebt sich bei der unterdrückten Gruppe und eröffnet den Individuen neue Möglichkeiten der Identifikation. Hybridität funktioniert hier als eine Transitzone für Identifikationen, in der Differenz ohne übernommene Hierarchie möglich ist (Bhabha 2000: 5). Hybridität bedeutet nicht, wie häufig fälschlicherweise angenommen wird, lediglich die gemischte ethnische und/oder kulturelle Formation. Letztere ergibt sich in einem Einwanderungsland wie Deutschland ohnehin von selbst. Hybridität bezieht sich insbesondere auf die kulturelle Logik der Übersetzung, die sich inmitten asymmetrischer Konstellationen seit Beginn des europäischen Globalisierungsprojekts in Form von ,westlicher Moderne vs. Rest der Welt“ (Hall 1996) ergibt (Hall 2004: 208). Der niemals endende Prozess der Re-Signifikation ist geprägt von schmerzhaften, auseinandersetzungsfreudigen und nicht leicht durchschaubaren Energien und Vorhaben, weil durch ihn Kulturen gezwungen werden, eigene Normen und moralische Werte nеu zu fassen. Am Konzept der Re-Signifikation ist erkennbar, dass die postkoloniale Perspektive weder "negative“ noch „positive“ Narrative über eingewanderte Menschen ins Leben ruft oder verteidigt, 
sondern der Beschreibung und Analyse jener neuartigen, durch kulturelle Übersetzung initiierten Formationen dient. Letztere können daher nur in medias res aufgedeckt werden, sind sie doch kein Bestandteil von ,Tradition“ oder „Moderne“ (ebd.: 339). Die aus der spannungsvollen Ambivalenz neu entstandene Formation wird als „Überlebensstrategie“ (ebd.: 257) begriffen, welche die eindimensionale Polarisierung von Herrscher und Beherrschtem ${ }^{14}$ zerfallen $^{2}$ lässt (Bhabha 1990: 220).

Im liminalen Raum Asyl verschwimmen die Grenzen in Bezug auf eindeutige Zuordnungen; im Vergleich zum Herkunftskontext herrschen bei Maryams Großfamilie etwa nicht mehr eindeutige Positionierungen in Kopftuchfragen vor. Zwar ist die Mimikry in diesem Fall mit Gewalt verbunden, doch eröffnen sich selbst hier durch die (Schein-)Freiheit in den Episoden außerhalb der eigenen vier Wände für die Betroffenen neue Möglichkeiten, sich vom Machtanspruch ihrer Männer zu befreien. Versteht man solche Dynamiken als Bhabha'sche Widerstandsformen, die auf die Prozessualität der kulturellen Differenz einwirken, stellt sich der Widerstand nicht bloß als eine Antithese oder ein Gegendiskurs dar; er

„,ist weder zwangsläufig ein politisch motivierter oppositioneller Akt, noch stellt er einfach die Negation oder den Ausschluß des ,Inhalts“ einer anderen Kultur als einer einmal wahrgenommenen Differenz dar. Er ist das Resultat einer Ambivalenz, die innerhalb der Erkenntnisregeln der dominanten Diskurse produziert wird, während sie die Zeichen kultureller Differenz artikulieren [...]“ (Bhabha 2000: 163).

Das Beispiel illustriert, dass Mimikry als unbewusste Form des kollektiven Widerstands verstanden wird. Zudem erinnert Maryam an die ,Sprachlosigkeit der Subalternen" (vgl. Abschn. 4.4). Maryam steht doppelt im Schatten. Sie ist bereits die geknechtete „Andere“, doch unter ,den Anderen“ wird sie als Frau zusätzlich unterdrückt. Die Schlussfolgerung, sie sei unfähig, zu sprechen, scheint zunächst bestätigt. Die intersektionale Verknechtung lässt ihre Subjektivität noch mehr verstummen, doch nur deshalb, weil das „Hören“ in Maryams Umfeld hegemonial (patriarchal) strukturiert ist. Ihre Repräsentation besteht daher zunächst aus einer Tarnmaske, denn eine eigene Stimme ist ihr verwehrt. Selbst wenn man sie zu Beginn ihres Aufenthalts in Deutschland sprechen ließe, wären die Fesseln des Patriarchats zu stark,

\footnotetext{
${ }^{14}$ Die Hegel'sche Denkfigur „Herr und Knecht“ ist aus diesem Blickwinkel betrachtet lediglich als Werkzeug zur Dekonstruktion festgefahrener Narrative und nicht als absolute, unbewegliche Konstellation zu verstehen.
} 
als dass eine authentische Stimme zu vernehmen wäre. Sie befürchtet die Ausgrenzung und Verachtung ihrer afghanischen Mitmenschen, weil sie sich trennen und emanzipieren möchte. Es dauert Monate, bis Maryam erst durch eine Fremdeinwirkung (Sohn) in der Lage ist, sich vorsichtig vom Ehemann zu lösen. Damit weist sie althergebrachte Reaktions- und Emotionsmuster zugunsten einer „moderneren“ Sichtweise zurück und reflektiert darüber im Zusammenhang mit dem Tragen des Kopftuches. Im neuen kulturellen Kontext haben Freiheit, die Gleichberechtigung zwischen Mann und Frau sowie die generelle soziale Ordnung ${ }^{15}$ einen viel höheren Stellenwert als noch im Ausgangskontext der Flucht. Die Vorstellung, dass eine gerechte und friedliche Gesellschaft ausschließlich in einem islamisch regierten Land möglich sei, hat sich beispielsweise nun vollständig im Denken Maryams aufgelöst. Sie führt diese Fehlvorstellung auf die Indoktrinierung des Mullah-Regimes zurück. Nun sei sie von der Überlegenheit der Demokratie, wie sie im Westen ausgeübt werde, überzeugt. Versteht man Emotionen als Botschaften des Selbst, so spürt sie durch ihre Emotionen im Ankunftskontext die Notwendigkeit der Neupositionierung seiner Sitten- und Moralvorstellungen. In der Reflexion über das Tragen des Kopftuchs realisiert Maryam, dass sich ihre ursprüngliche Haltung in diesem Punkt gewandelt hat. Dies macht sich auch darin bemerkbar, dass sie das Kopftuch weniger gewissenhaft trägt - nun ist ein kleiner Teil der Haare zu sehen - und sie diesbezüglich deutlich unbekümmerter ist. Doch die besonders rigide Art in Maryams sozialem Umfeld, Frauen zu stigmatisieren, die sich emanzipieren möchten, hindert sie daran, vollends aus ihrer alten Rolle herauszutreten. Kulturelle und religiöse Elemente, die teils nicht nachvollziehbar sind für mich, dienen so ebenfalls zur Rechtfertigung ihres Zustandes, der als Stillstand gedeutet wird. An der Frage, ob das Kopftuch entfernt werden soll oder nicht, arbeitet Maryam ihre unbewusste Verunsicherung, die im Ankunftskontext zunimmt, ab. Obwohl sie in Deutschland zum ersten Mal fühlt, welche neuen Möglichkeiten sich durch das Leben ohne Kopftuch ergeben, ist sie unfähig, wirkliche Freude zu empfinden. Hierin zeigt sich eine weitere Dimension ihrer emotionalen Verfassung. Die Verkennung ihrer Persönlichkeit als intelligente und offenbar höchst widerstandsfähige Frau und die daraus hervorgehenden unerfüllten Wünsche und Impulse im Heimatkontext sowie ihre höchste Zielsetzung, ein freies Leben als moderne Frau im Ankunftskontext zu führen, konstituieren ihre zwiespältige

\footnotetext{
${ }^{15}$ Gemeint ist die Ordnung und Regelmäßigkeit im öffentlichen Leben Deutschlands, die sie oft anmerkt, z. B. die Gewaltentrennung, die Unbestechlichkeit der Staatsorgane und die daraus entstehende sichere Atmosphäre des sozialen Lebens.
} 
Situation: In ihrem Verhalten und insbesondere in ihren emotionalen Ausdrucksweisen spiegelt sich daher ihr Uneins-Sein wider. Das zeigt sich beispielsweise, wenn sie für ihren Sohn die starke Mutter spielt, die alles hinnimmt und sich aufopfert, während sie immer wieder aus dieser Rolle herausschlüpft und sich in unglaublich devoten Aussprüchen bzw. Verhaltensweisen verliert, die das orientalische ta'arof-Spiel $^{16}$ weit übersteigen. In ihrem narrativen Projekt (Katz III) inszeniert sie unterschiedliche Facetten dieser beiden gegenteiligen Rollen mit den entsprechenden affektiven Bezugnahmen. Dennoch hält sie an dem erlebten Gefühl fest, das sie als „großartig“ bezeichnet. Es scheint, als ob sie es wie einen Schatz in den Tiefen ihres Geistes vergräbt, um darauf zurückzukommen, wenn sie alle Hürden der Institution Asyl sowie andere, selbstauferlegte Barrieren - etwa die zusätzliche Wartezeit, bis sie sich offiziell als geschieden betrachtet - gemeistert haben wird. Häufig lässt Maryam erkennen, wie sehr sie sich wünscht, eine „moderne Frau“ zu sein, was ihr - ihrer Ansicht nach - jedoch nicht gelingt. Dann schweift sie ab und verweilt in Gedanken. Sie spricht ohnehin sehr zurückhaltend, doch in Momenten, in denen dieses Thema zur Debatte steht, wird ihre Stimme immer leiser, bis sie nicht mehr zu verstehen ist. Spreche ich sie in dieser Situation an, schreckt sie zusammen und hält sich den Kopf fest. Gleichzeitig winkt sie bereits mit einer Hand ab, als wolle sie mir bedeuten, ihr Zustand sei gar nicht so schlimm, wie es vielleicht wirken mag - eine typische Ausdrucksweise in Maryams Verhalten. Im sozialen Drama, das sie vor und mit mir entfaltet, stehen demnach zwei gegenpolige emotionale Erscheinungen im Zentrum: Auf der einen Seite ist dies die exponierte Sittsamkeit Maryams, das sie mit entsprechenden Affekten und Ausdrucksweisen aufführt. Hierin spiegelt sich die Hilfsbedürftigkeit und Machtlosigkeit wider. Auf der anderen Seite befindet sich Maryams Sehnen nach dem Leben als „,moderne Frau“. Dies lässt Wut aufkeimen. Zunächst wird dies in der Transformation ihres Körpers durch die totalitären Merkmale im Ankunftskontext ausgelöst. Das unerträglich lange Warten und die existenzielle Angst beeinträchtigen Maryams Handlungsfähigkeit. Ihre psychische Integrität wird so stückweise zerstört. Die daraus folgende sinnliche Metamorphose (Katz I) ermöglicht den Ausdruck ihrer individuellen Gefühle. Zur Wut trägt ebenfalls der Umstand bei, dass sie fortwährend verletzt wurde, hauptsächlich aufgrund der afghanischen Herkunft und darüber hinaus aufgrund ihres Geschlechts. Angesichts dieser Verletzungen keimt die Emotion Scham in ihr auf, die sich erst nach vielen Jahren in Wut verwandelt. Man sollte nicht aus den Augen verlieren, dass rassistische und sexistische Übergriffe auf Afghan*innen

\footnotetext{
${ }^{16}$ Besondere Form der Höflichkeit im Orient (siehe Fallbeispiel Nr. 2).
} 
auf der Agenda der Teheraner Slums ganz oben stehen. Hinzu kommt die Gleichgültigkeit ihres Mannes, die sich im Laufe der Jahre in Hass transformiert. Er wird handgreiflich und verletzt sie zunehmend mit seinen Verbalattacken. Freundschaften konnte sie nicht entwickeln, nur Bekanntschaften, mit denen sie ihre dunklen Geheimnisse lieber nicht teilte, um wenigstens eine heile Welt außerhalb ihres Heims zu haben. Sie wurde nie beachtet, obwohl sie sich um Anerkennung bemühte, etwa durch ihre bemerkenswerten Leistungen an der Universität. Die erwähnte Missachtung setzt sich fort. Im Zielland ihrer Flucht wird Maryam weiterhin durch die Institution Asyl in ihrem Fortkommen behindert. Die sinnliche Metamorphose (Katz I) und die Resonanz der sozialen Interaktionen (Katz II) spornen sie dazu an, eine Narration zu entwerfen, die es erlaubt, einen Ausweg aus ihrem Dilemma zu finden. Denn Wut aktiviert, mobilisiert und setzt Kräfte frei, die sie nun unbedingt benötigt (Handlungstendenz, Emotionsmodell Baustein IV). Ihr bewunderndes Verlangen nach dem Leben als moderne Frau, die sich nicht um patriarchale Sittengesetze kümmert, ist ebenfalls ein antreibender Faktor. Ihr System - sowohl körperlich als auch psychisch - teilt ihr indes mit, dass der Dissens aufgelöst werden soll. Körperliche Metamorphosen in Form der genannten Symptome enthalten demnach relevante Informationen für den Emotionen erlebenden Menschen. Die individuelle Dimension der Emotion Wut geht aus der situationstranszendierenden Bedeutung hervor, die Maryam den Auslösern der Wut zuschreibt. Die spezifischen Auslöser, etwa die Zeitvergeudung, versteht sie mithin als „situationstranszendierende Herausforderungen“ (vgl. Dietz 2012: 36) an das eigene Selbst. In diesem Lichte „,widerfährt“ ihr nicht bloß Wut und Verzweiflung, die dominierenden Emotionen verwandeln sich vielmehr durch den komplexen Prozess im Ankunftskontext (sinnliche Metamorphose (Katz I), soziale Interaktionen (Katz II) sowie daraus entspringende Bedeutungszuschreibungen) zu einer aktiven und kreativen Handlungspraxis, mittels derer Maryam eine spezifische Narration (Katz III) konstruiert. Jetzt im Ankunftskontext scheint die Zeit zu sein, in der sie die Überforderungen und Unterdrückungen ihres Gefühlslebens der letzten anderthalb Jahrzehnte, vor allem jedoch den dahinterstehenden gesellschaftlichen Zwang ihres Ausgangskontextes infrage stellen und im wahrsten Sinne des Wortes loslassen kann. Wut bildet den Motor für diese Entwicklung. Ihr Drang zur Sittsamkeit und die ständigen Reflexionen über das Kopftuch können als erste Schritte einer Auseinandersetzung mit Fragen gedeutet werden, die für ihr Ziel, eine emanzipierte Frau zu sein, von Relevanz sind. Widersprüchliche Emotionen und die Unklarheit gewisser Aspekte der „modernen“ Lebensauffassung erlauben ihr vermittels Selbstreflexion eine graduelle Distanzierung von ihrer alten unterlegenen Rolle zugunsten derjenigen einer modernen Heldin. Die Unterwerfung Maryams unter die anrufende Macht 
des dominanten Diskurses im Ankunftskontext bewirkt ihre „Subjektwerdung“ (Butler 2001: 28). Maryams Handlungsfähigkeit wird ,paradoxerweise“ (ebd.: 8) durch die vorgängige Macht ermöglicht, ohne von dieser vollständig begrenzt zu werden. Der erwähnte dominante Diskurs wird einerseits von weiteren, in dieser Fallstudie herausgearbeiteten, totalitären Merkmalen der Institution Asyl - etwa dem übermäßigen Warten und dem entsprechenden Gefühl der Zeitvergeudung, dem Suggerieren eines Zustandes, der einem „Missgeschick“ oder „Scheitern“ gleicht, der Kontrolle des sozialen Raums, der permanenten Produktion von Angst und Unsicherheit sowie Eingriffen in die psychische Integrität - und andererseits von patriarchalen Dynamiken ihres sozialen Umfeldes - Mimikry ihrer Großfamilie und Stigmatisierung durch afghanische Community - geprägt. Maryam ist zwar in der Diskursstruktur der Institution Asyl eingebettet, doch bringt sie durch ihre Narration gemäß Katz selbst ihre Emanzipation und damit ihre Subjektwerdung hervor. Die paradoxe Ambivalenz der Subjektivation offenbart sich in der vorliegenden Interpretation daran, dass sich Maryams Handlungsfähigkeit als Effekt ihrer Unterwerfung zeigt (vgl. Abschn. 4.6). Sie erhält ihre Position in der herrschenden diskursiven Ordnung, indem sie sich den normierenden Zwängen unterordnet. Maryams emanzipatorische Bestrebungen ließen sich nicht adäquat nachvollziehen, wenn Macht und Subjekt als getrennte Entitäten gedacht würden. Auch wird an diesem Beispiel deutlich, inwiefern Maryam genötigt wird, in Kategorien und Begriffen nach Anerkennung zu suchen, die sie selbst nicht hervorgebracht hat (ebd.: 25).

Die Kombination der hier verwendeten theoretischen Ansätze erlaubt es, widersprüchlich erscheinende Emotionen als relational einander zugeordnete Pole zu begreifen, die in permanenter Wechselwirkung und gegenseitiger Kontrastierung das Befinden Maryams konstituieren. Durch die ängstliche Art, sich nur allmählich und mit erfundenen Wartezeiten ,endgültig“ von ihrem Mann zu trennen, gibt sie zugleich ihrer Vergangenheit, insbesondere der Zeit mit ihrem Mann, eine Bedeutung und verzögert so ihre Emanzipation. Ihre Angstgefühle garantieren zudem eine vorsichtige Anpassung in der Zukunft. Die selbstaufgebürdete Wartezeit kann als eine Strafe gedeutet werden, die sie zu ertragen hat, um das ersehnte Ziel als ultimative Belohnung zu erreichen. Die Zeit, welche die Reflexion und Neubewertung ihrer Situation bedarf, wird mithilfe der Inszenierung ihres persönlichen Dramas ausgefüllt. Von diesem Standpunkt aus eignet sich das existenzielle Gefühl (Ratcliffe) ihres Dramas, nämlich das des Gescheitert-Seins, ebenfalls für die Deutung der Selbstbestrafung. Der Tiefpunkt einer gescheiterten Person wird letztlich zur Wiege ihrer Auferstehung als Heldin.

Dieses Fallbeispiel illustriert weitere Merkmale der totalen Institution Asyl (siehe weiter oben), die das Selbst des gefangenen Menschen angreifen. Besonders unter den Bedingungen der sozialräumlichen Enge innerhalb der 
Institution Asyl repräsentiert das maßlose Warten eine Offensive auf das Selbst Maryams. Die totalitären Merkmale müssen von ihren Funktionen, die sich durchweg als repressiv herausgestellt haben, unterschieden werden. Ähnlich wie bei anderen bereits besprochenen Charakteristika der Institution Asyl, beispielsweise dem Looping-Effekt oder der Fragehoheit/Rechtfertigungsangst, fungieren sie als Kommunikation und Demonstration von Macht und etablieren unter anderem Ein- und Ausgrenzungsmechanismen im Ankunftskontext. Die herausgestellten Merkmale der Institution Asyl stoßen Dynamiken an, die Asylbewerber*innen isolieren und stigmatisieren, weshalb sie als „soziale Folterwerkzeuge“ verstanden werden. Deutlich wurde dies vor allem durch die Pathologisierung der hier betrachteten Person, die ihren Aufenthalt nur durch ein Attest über eine schwerwiegende psychische Erkrankung sichern kann. Durch den Druck, die eigene psychische Krankheit attestieren zu müssen, um die Abschiebung zu verhindern, wird das „Missgeschick“, ein „abgelehnter Flüchtling“ zu sein, vergrößert. Denn ein psychisch kranker und ,abgelehnter Flüchtling" stellt ein weit unliebsameres Missgeschick und zur selben Zeit eine zusätzliche Diskriminierungsdimension dar. Die Einschränkung der Handlungsmächtigkeit eines ausgegrenzten und unterdrückten Menschen wird erneut durch die Vergabe von Psychopharmaka verstärkt.

Open Access Dieses Kapitel wird unter der Creative Commons Namensnennung 4.0 International Lizenz (http://creativecommons.org/licenses/by/4.0/deed.de) veröffentlicht, welche die Nutzung, Vervielfältigung, Bearbeitung, Verbreitung und Wiedergabe in jeglichem Medium und Format erlaubt, sofern Sie den/die ursprünglichen Autor(en) und die Quelle ordnungsgemäß nennen, einen Link zur Creative Commons Lizenz beifügen und angeben, ob Änderungen vorgenommen wurden.

Die in diesem Kapitel enthaltenen Bilder und sonstiges Drittmaterial unterliegen ebenfalls der genannten Creative Commons Lizenz, sofern sich aus der Abbildungslegende nichts anderes ergibt. Sofern das betreffende Material nicht unter der genannten Creative Commons Lizenz steht und die betreffende Handlung nicht nach gesetzlichen Vorschriften erlaubt ist, ist für die oben aufgeführten Weiterverwendungen des Materials die Einwilligung des jeweiligen Rechteinhabers einzuholen.

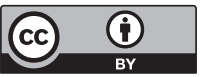

